\section{Generalised lymphangiectasia: pulmonary presentation in an adult}

\author{
J E S White, D Veale, D Fishwick, \\ L Mitchell, P A Corris
}

\begin{abstract}
A 25 year old man presented with dyspnoea and was found to have generalised, but predominantly pulmonary, lymphangiectasis without gastrointestinal symptoms. This is an unusual presentation of a disorder previously diagnosed only in childhood.
\end{abstract}

(Thorax 1996;51:767-768)

Keywords: lymphangiectasia, adult.

Pulmonary lymphangiectasis was first described by Virchow over 100 years ago $^{1}$ and can be classified as primary, secondary, or part of a generalised lymphatic abnormality..$^{2-4} \mathrm{We}$ describe a patient who presented in adult life with generalised lymphangiectasis and predominantly pulmonary disease which has not been reported previously.

\section{Case report}

A 25 year old man presented with a three month history of breathlessness on exertion. He had suffered from asthma from six to 13 years of age and had no other illnesses. There was no significant family history and examination revealed no abnormal physical findings. A chest radiograph showed widespread interstitial shadowing throughout both lung fields. Echocardiography was normal and, in particular, there was no abnormality of the proximal pulmonary veins or left atrium. An open lung biopsy specimen revealed a small amount of clear yellow pleural fluid. The surface of the lung was inflamed and the underlying lung was stiff; the lymphatic vessels were markedly distended. Light microscopic examination showed wide interlobular septae with marked interstitial oedema. A considerable proliferation of dilated lymphatic vessels staining positively for elastin was seen $-\mathrm{a}$ probable consequence of lymphatic obstruction outside the lungs.

Two months later he presented with severe dyspnoea due to a large right sided pleural effusion which was aspirated with symptomatic relief. He remained well enough to continue playing sports for four years but then gradually developed increasing breathlessness over a period of six years culminating in readmission.
On examination he looked unwell and was cyanosed and breathless on minimal exertion. He had a swollen oedematous right leg with cutaneous discolouration. There was no palpable lymphadenopathy. Examination of the chest revealed dullness to percussion at the right base with reduced breath sounds and widespread wheezes in all other zones. Investigation showed normal haemoglobin, mild thrombocytopenia (platelets $106 \times 10^{9} / 1$ ) and a raised white blood cell count of $14 \times 10^{9} / 1$ with $88 \%$ neutrophils. Blood gas tensions breathing room air showed a $\mathrm{PaO}_{2}$ of $8.6 \mathrm{kPa}$ and a $\mathrm{PaCO}_{2}$ of $7 \cdot 7 \mathrm{kPa}$. Spirometric tests gave a forced expiratory volume in one second $\left(\mathrm{FEV}_{1}\right)$ of 0.71 ( $15 \%$ predicted) and a vital capacity (VC) of 1.71 ( $29 \%$ predicted). Chest radiography showed pleural calcification with a large pleural effusion on the right and diffuse interstitial shadowing throughout the lung fields (figure). A computed tomographic scan of the chest showed massive mediastinal lymphatic dilatation surrounding and encasing the mediastinal structures and appearing to extend out into the lung fields. These appearances extended down into the abdomen and around the coeliac axis. The possibility of lymphatic obstruction due to enlarged lymph nodes was considered. Mediastinoscopy was attempted but the large, tense, dilated lymphatic vessels had the appearance of dilated veins and the procedure was abandoned for fear of haemorrhage. A right explorative thoracotomy was subsequently performed and a loculated pleural collection was drained yielding dark caseous material and altered blood. The surrounding thick and calcified pleura was decorticated. Postoperative treatment with nebulised bronchodilators and oral corticosteroids was followed by an improvement in lung function with $\mathrm{FEV}_{1}$ rising to $1.5 \mathrm{l}$, VC to $2.6 \mathrm{l}$, attributable in part to an increase in lung volume after surgery but also to a significant asthmatic component confirmed by diurnal variability in peak

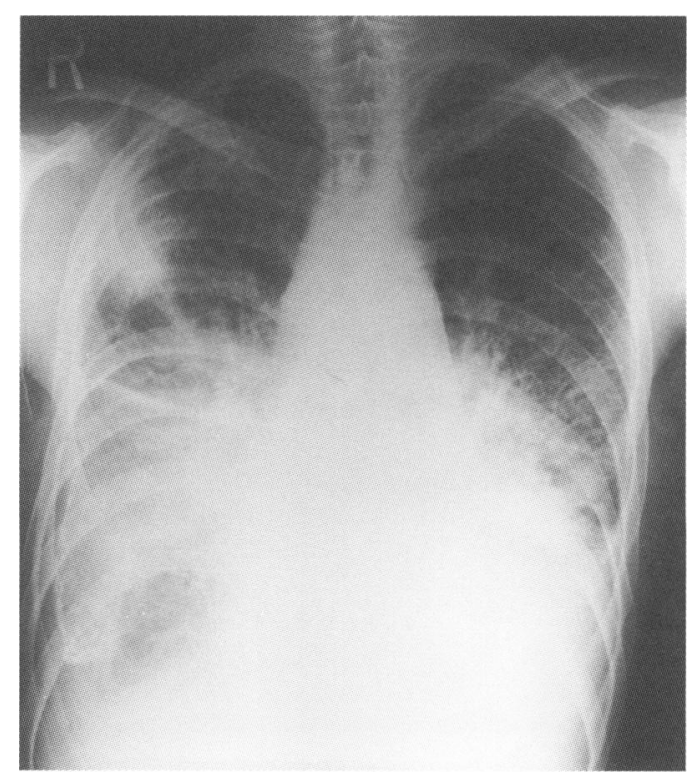

Posteroanterior chest radiograph showing pleural shadowing and calcification on the right and diffuse interstitial shadowing throughout both lung fields. 
flow. In view of the concerns raised at surgery, central venography was performed via a femoral venous approach which showed that the subclavian and iliac veins and the inferior and superior venae cavae were normal. Follow through of contrast media provided images of the pulmonary arteries and veins which were also normal. A lymphangiogram was performed using a standard pedal approach and showed grossly distended lymphatic vessels in the upper abdomen and in the mediastinum, up to $1 \mathrm{~cm}$ in diameter with no evidence of obstruction or other apparent cause.

Asthma treatment with high dose inhaled corticosteroids via a large volume spacing device led to an improvement in his clinical condition until the development of a large left chylothorax which was successfully treated with aspiration and chemical pleurodesis. A diet rich in medium chain triglycerides and high in protein intended to reduce the lymphatic flow from the small intestine has prevented recurrence of chylothorax.

\section{Discussion}

Pulmonary lymphatic abnormalities can be categorised as lymphangiomas, lymphangioleiomyomatosis or lymphangiectasis.

The causes of pulmonary lymphangiectasis have been divided into (1) primary congenital lymphatic abnormalities, (2) those secondary to pulmonary venous obstruction either due to venous or cardiac morphological abnormalities, and (3) those which are part of a generalised lymphatic abnormality.

Our patient did not have the classical congenital form of lymphangiectasis as this presents in infancy and is rapidly fatal. He did not have any cardiac disorder and thus conforms most closely to the pattern of generalised lymphangiectasis with lymphatic abnormalities above and below the diaphragm. He had an unusual presentation, however, as all previous cases with generalised lymphangiectasis have presented in infancy, were associated with hemihypertrophy (that is, enlargement of one side of the body), peripheral oedema, and had intestinal involvement. ${ }^{67}$ Our patient presented at the age of 25 years, had little limb oedema, and had never had gastrointestinal symptoms or features of malabsorption. He does, however, have asthma which has previously been reported as a feature in two patients with generalised lymphangiectasis ${ }^{78}$ aged 6.5 and 11 years, respectively. One had dilated pulmonary lymphatic vessels proven on biopsy. ${ }^{8}$ Pulmonary involvement appeared to be less severe in these patients and was associated with a much better prognosis. Previously reported cases of pulmonary lymphangiectasis in adults had localised disease only which was successfully treated by surgical removal of the affected lobe. $^{3}$

Treatment of generalised lymphangiectasis and chylothorax is difficult but dietary control has been shown to be of some benefit. ${ }^{910}$ It has been suggested that low fat intake reduces lymphatic drainage from the small intestine. ${ }^{10}$ Surgical procedures such as a LaVeen shunt from the left pleural space to the inferior vena cava have been used in rare instances with some success. ${ }^{10}$ The patient we report is unlike any previously described. He bears closest resemblance to the group with generalised lymphangiectasis. He has been spared significant gastrointestinal features and this may partly explain his late presentation. We conclude that pulmonary lymphangiectasis may have a more benign course than previously described.

1 Virchow R. Gesammelte Abhandlungen zur Wissenschaftlichen Medicin. Frankfurt: Meidinger Sohn \& Co, 1856:982.

2 Noonan JA, Walters LR, Reeves JT. Congenital pulmonary lymphangiectasis. Am $\mathcal{F}$ Dis Child 1970;120:314-9.

3 Wagenaar SJ, Swierenga J, Wagenvoort CA. Late presentation of primary pulmonary lymphangiectasis. Thorax 1978;33:791-5.

4 Levine C. Primary disorders of the lymphatic vessels - a unified concept. F Pediatr Surg 1989;24:233-40.

5 Kelso JM, Kerr DJ, Lie JT, Sachs MI, O'Connel EJ. Unusua diffuse pulmonary lymphatic proliferation in a young boy. Chest 1991;100:556-60.

6 Frank J, Piper PG. Congenital pulmonary cystic lymphangiectasis. $\mathscr{F} A M A 1959 ; 171: 1094-8$.

7 Mann TP. Hemihypertrophy left side of body: congenital lymphatic oedema of left arm: radiological enlargement lymphatic oedema of left arm: radiological en
of heart shadow. Proc $R$ Soc Med 1955;48:330.

8 Shubert et al. Cited in Noonan JA, Walters LR, Reeves JT. Congenital pulmonary lymphangiectasis. Am $\mathcal{f}$ Dis Child 1970;120:314-9.

9 Colpu L, Emri S, Selcuk ZT, Kaloncu F, Balkanci F, Sahin AA, et al. Life threatening chylous pleural and pericardial effusion in a patient with Behcet's syndrome. Thorax 1992 47:64-5.

10 Lester LA, Rothberg RM, Krantman HJ, Shermeta DW. Intestinal lymphangiectasia and bilateral pleural effusions: effects of dietary therapy and surgical intervention on immunologic and pulmonary parameters. 7 Allergy Clin immunol 1986;78:891-7. 